\title{
JORDAN *-DERIVATIONS OF STANDARD OPERATOR ALGEBRAS
}

\author{
PETER ŠEMRL
}

(Communicated by Palle E. T. Jorgensen)

\begin{abstract}
Let $H$ be a real or complex Hilbert space, $\operatorname{dim} H>1$, and $\mathscr{B}(H)$ the algebra of all bounded linear operators on $H$. Assume that $\mathscr{A}$ is a standard operator algebra on $H$. Then every additive Jordan *-derivation $J: \mathscr{A} \rightarrow$ $\mathscr{B}(H)$ is of the form $J(A)=A T-T A^{*}$ for some $T \in \mathscr{B}(H)$.
\end{abstract}

Let $\mathscr{A}$ be a real or complex $*$-algebra and $\mathscr{A}_{1}$ any subalgebra of $\mathscr{A}$. An additive (linear) mapping $D: \mathscr{A}_{1} \rightarrow \mathscr{A}$ is called an additive (linear) Jordan derivation if $D\left(a^{2}\right)=a D(a)+D(a) a$ for all $a \in \mathscr{A}_{1}$. An additive (real-linear) Jordan $*$-derivation $J: \mathscr{A}_{1} \rightarrow \mathscr{A}$ is defined as an additive (real-linear) mapping satisfying $J\left(a^{2}\right)=a J(a)+J(a) a^{*}$ for all $a \in \mathscr{A}_{1}$. It is easy to verify that for an arbitrary element $b \in \mathscr{A}$ the mapping $D_{b}: \mathscr{A}_{1} \rightarrow \mathscr{A}$ defined by $D_{b}(a)=$ $a b-b a\left(D_{b}(a)=a b-b a^{*}\right)$ is a Jordan derivation (Jordan *-derivation).

The study of Jordan *-derivations has been motivated by the problem of the representability of quasi-quadratic functionals by sesquilinear ones (for the results concerning this problem we refer to $[5,7,9-11])$. It turns out that the question of whether each quasi-quadratic functional is generated by some sesquilinear functional is intimately connected with the structure of Jordan *-derivations [7,9].

Let $H$ be a real or complex Hilbert space. By $\mathscr{B}(H)$ we mean the algebra of all bounded linear operators on $H$. We denote by $\mathscr{F}(H)$ the subalgebra of bounded finite rank operators. We shall call a subalgebra $\mathscr{A}$ of $\mathscr{B}(H)$ standard, provided $\mathscr{A}$ contains $\mathscr{F}(H)$. It is easy to see that $\mathscr{F}(H)$ is a prime ring; that is, $A, B \in \mathscr{F}(H)$ and $A \mathscr{F}(H) B=\{0\}$ imply $A=0$ or $B=0$.

Assume that $H$ is an infinite-dimensional Hilbert space. Let $\mathscr{A}$ be a standard operator algebra on $H$. Suppose that $D: \mathscr{A} \rightarrow \mathscr{B}(H)$ is an additive Jordan derivation. Every finite rank operator is a linear combination of idempotent operators of rank one. If $P$ is an idempotent operator of rank one and $\lambda=\mu^{2}$ is a scalar, then $D(\lambda P)=\mu(P D(\mu P)+D(\mu P) P)$ has rank at most two. Thus, $D$ maps $\mathscr{F}(H)$ into itself. Using the result of Herstein [3], which states that all additive Jordan derivations of prime rings of characteristic different from two are derivations, we infer that $D$ satisfies $D(A B)=A D(B)+D(A) B$ for all pairs $A, B \in \mathscr{F}(H)$. It follows from [8] that there exists $T \in \mathscr{B}(H)$

Received by the editors May 26, 1992.

1991 Mathematics Subject Classification. Primary 47B47, 47D25.

This work was supported by the Research Council of Slovenia. 
such that

$$
D(A)=A T-T A
$$

for all finite rank operators $A$. Linearizing the equation $D\left(A^{2}\right)=A D(A)+$ $D(A) A$ we get $D(A B+B A)=A D(B)+B D(A)+D(A) B+D(B) A$. Together with (1) this yields that

$$
B(D(A)-A T+T A)+(D(A)-A T+T A) B=0
$$

holds for all $A \in \mathscr{A}, B \in \mathscr{F}(H)$. Consequently, we have $D(A)=A T-T A$ for every $A \in \mathscr{A}$. Thus, every additive Jordan derivation $D: \mathscr{A} \rightarrow \mathscr{B}(H)$ is of form (1) for some $T \in \mathscr{B}(H)$. The assumption that $H$ is infinite dimensional is indispensable in this statement [8].

It is the aim of this note to obtain a similar result for additive Jordan *-derivations. More precisely, we shall prove the following result:

Theorem. Let $H$ be a real or complex Hilbert space, $\operatorname{dim} H>1$, and let $\mathscr{A}$ be a standard operator algebra on $H$. Suppose that $J: \mathscr{A} \rightarrow \mathscr{B}(H)$ is an additive Jordan *-derivation. Then there exists a unique linear operator $T \in \mathscr{B}(H)$ such that $J(A)=A T-T A^{*}$ holds for all $A \in \mathscr{A}$.

Remark. Two special cases of this result have been already proved-the case when $\mathscr{A}=\mathscr{B}(H)[6]$ and the case when $H$ is a complex Hilbert space and $\mathscr{A}$ is the algebra of all compact linear operators [1]. In this general setting we use a completely different approach as in $[1,6]$.

Proof. Let us denote by $J_{1}$ the restriction of $J$ to the ideal $\mathscr{F}(H)$. We define a mapping $\phi: \mathscr{F}(H) \rightarrow \mathscr{B}(H \oplus H)$ by

$$
\phi(A)=\left(\begin{array}{cc}
A & J_{1}(A) \\
0 & A^{*}
\end{array}\right) .
$$

Clearly, $\phi$ is an additive Jordan homomorphism; that is, $\phi$ is additive and $(\phi(A))^{2}=\phi\left(A^{2}\right)$ holds for all finite rank operators $A$. It should be mentioned that relation (2) is a variation of a standard connection between linear derivations and algebra homomorphisms (see [2]). Since $\mathscr{F}(H)$ is a locally matrix algebra, by a result of Jacobson and Rickart [4], $\phi=\varphi+\psi$, where $\varphi: \mathscr{F}(H) \rightarrow \mathscr{B}(H \oplus H)$ is a ring homomorphism and $\psi: \mathscr{F}(H) \rightarrow \mathscr{B}(H \oplus H)$ is a ring antihomomorphism. We have

$$
\operatorname{Im} \phi \subset\left\{\left(\begin{array}{cc}
X & Y \\
0 & W
\end{array}\right) \in \mathscr{B}(H \oplus H): X, Y, W \in \mathscr{B}(H)\right\} .
$$

It follows that $\varphi$ and $\psi$ are of the form

$$
\varphi(A)=\left(\begin{array}{cc}
\varphi_{1}(A) & \varphi_{2}(A) \\
0 & \varphi_{3}(A)
\end{array}\right), \quad \psi(A)=\left(\begin{array}{cc}
\psi_{1}(A) & \psi_{2}(A) \\
0 & \psi_{3}(A)
\end{array}\right)
$$

where $\varphi_{1}, \varphi_{3}$ are additive homomorphisms on $\mathscr{F}(H), \psi_{1}, \psi_{3}$ are additive antihomomorphisms on $\mathscr{F}(H)$, and the equations $\varphi_{1}(A)+\psi_{1}(A)=A$ and $\varphi_{3}(A)+\psi_{3}(A)=A^{*}$ are valid for all $A \in \mathscr{F}(H)$. Pick an idempotent $P$ on $H$ of rank one. Then $P$ is the sum of the idempotents $\varphi_{1}(P)$ and $\psi_{1}(P)$; therefore, we have that either $\varphi_{1}(P)=0$ or $\psi_{1}(P)=0$. Thus, at least one of $\varphi_{1}$ and $\psi_{1}$ has a nonzero kernel. Since the kernels of homomorphisms and antihomomorphisms are ideals and since the only nonzero ideal of $\mathscr{F}(H)$ is 
$\mathscr{F}(H)$ itself, we have $\varphi_{1}=0$ or $\psi_{1}=0$. As a consequence we get $\psi_{1}=0$ and $\varphi_{1}(A)=A$ for all $A \in \mathscr{F}(H)$. Similarly we show that $\varphi_{3}=0$. Thus, relations (3) can be rewritten as

$$
\varphi(A)=\left(\begin{array}{cc}
A & \varphi_{2}(A) \\
0 & 0
\end{array}\right), \quad \psi(A)=\left(\begin{array}{cc}
0 & \psi_{2}(A) \\
0 & A^{*}
\end{array}\right) .
$$

The mappings $\varphi$ and $\psi$ are an additive homomorphism and an additive antihomomorphism respectively, and consequently, $\varphi_{2}$ and $\psi_{2}$ are additive mappings satisfying

$$
\varphi_{2}(A B)=A \varphi_{2}(B)
$$

and

$$
\psi_{2}(A B)=\psi_{2}(B) A^{*}
$$

for all $A, B \in \mathscr{F}(H)$. Applying $J_{1}=\varphi_{2}+\psi_{2}, J_{1}\left(A^{2}\right)=A J_{1}(A)+J_{1}(A) A^{*},(4)$, and (5), one can see that $\varphi_{2}(A) A^{*}+A \psi_{2}(A)=0$ holds true for all $A \in \mathscr{F}(H)$. Linearizing this relation we get that $\varphi_{2}(A) B^{*}+\varphi_{2}(B) A^{*}+A \psi_{2}(B)+B \psi_{2}(A)=0$ for all $A, B \in \mathscr{F}(H)$. Replacing $B$ by $C B$ we obtain

$$
C\left(\varphi_{2}(B) A^{*}+B \psi_{2}(A)\right)+\left(\varphi_{2}(A) B^{*}+A \psi_{2}(B)\right) C^{*}=0
$$

for every finite rank operator $C$. Consequently, we have

$$
\varphi_{2}(A) B^{*}+A \psi_{2}(B)=0
$$

for all finite rank operators $A$ and $B$.

For any $x, y \in H$ we shall denote the inner product of these two vectors by $y^{*} x$, while $x y^{*}$ shall denote the rank one operator given by $\left(x y^{*}\right) z=\left(y^{*} z\right) x$. Every rank one operator can be written in this form. For every nonzero $x \in H$ we denote $L_{x}=\left\{x y^{*}: y \in H\right\} \subset \mathscr{F}(H)$. It follows from (4) that $\varphi_{2}$ is a linear mapping on $\mathscr{F}(H)$. Moreover, for every nonzero $x \in H$ we have $\varphi_{2}\left(L_{x}\right) \subset L_{x}$. Thus, we can find for every nonzero $x$ from $H$ a linear mapping $S_{x}: H \rightarrow H$ such that $\varphi_{2}\left(x y^{*}\right)=x\left(S_{x} y\right)^{*}$. For linearly independent vectors $x, u \in H$ and for an arbitrary vector $y \in H$ we have

$$
(x+u)\left(S_{x+u} y\right)^{*}=\varphi_{2}\left((x+u) y^{*}\right)=\varphi_{2}\left(x y^{*}\right)+\varphi_{2}\left(u y^{*}\right)=x\left(S_{x} y\right)^{*}+u\left(S_{u} y\right)^{*} .
$$

This yields that $S_{x}=S_{u}$. In the case that nonzero vectors $x$ and $u$ are linearly dependent, we find a vector $z$ from $H$ such that $x$ and $z$ are linearly independent. Then we have $S_{x}=S_{z}=S_{u}$. Hence, we have proved that there exists a linear operator $S: H \rightarrow H$ such that

$$
\varphi_{2}\left(x y^{*}\right)=x(S y)^{*} \text {. }
$$

One can verify using (5) that the mapping $\psi_{2}^{\prime}$ given by $\psi_{2}^{\prime}(A)=\left(\psi_{2}(A)\right)^{*}$ satisfies $\psi_{2}^{\prime}(A B)=A \psi_{2}^{\prime}(B)$. This yields the existence of a linear operator $T: H \rightarrow H$ such that

$$
\psi_{2}\left(x y^{*}\right)=-T y x^{*} .
$$

Replacing $A$ and $B$ in (6) by $x y^{*}$ and $u v^{*}$ respectively and applying (7), (8) we get that $(S y)^{*} v=y^{*} T v$ for all $v, y \in H$. It follows from the closed graph theorem that the operators $S$ and $T$ are bounded. Moreover, we have 
$S=T^{*}$. The equation $J_{1}=\varphi_{2}+\psi_{2}$ yields

$$
J(A)=A T-T A^{*}
$$

for every finite rank operator $A$.

Replacing $A$ by $A+B$ in $J\left(A^{2}\right)=A J(A)+J(A) A^{*}$, we get that

$$
J(A B)+J(B A)=A J(B)+B J(A)+J(A) B^{*}+J(B) A^{*}
$$

is valid for an arbitrary pair of operators $A, B$ from $\mathscr{A}$. Applying this relation with (9) we see that

$$
B\left(J(A)-A T+T A^{*}\right)+\left(J(A)-A T+T A^{*}\right) B^{*}=0
$$

holds true for all $A \in \mathscr{A}$ and all finite rank operators $B$. Thus, (9) is satisfied for all $A \in \mathscr{A}$. This completes the proof.

\section{REFERENCES}

1. M. Brešar and B. Zalar, On the structure of Jordan *-derivations, Colloq. Math. 63 (1992), 163-171.

2. P. R. Chernoff, Representations, automorphisms and derivations of some operator algebras, J. Funct. Anal. 12 (1973), 275-289.

3. I. N. Herstein, Jordan derivations of prime rings, Proc. Amer. Math. Soc. 8 (1957), 11041110.

4. N. Jacobson and C. Rickart, Jordan homomorphisms of rings, Trans. Amer. Math. Soc. 69 (1950), 479-502.

5. P. Šemrl, On quadratic functionals, Bull. Austral. Math. Soc. 37 (1988), 27-29.

6. __ On Jordan *-derivations and an application, Colloq. Math. 59 (1990), 241-251.

7. __ Quadratic functionals and Jordan *-derivations, Studia Math. 97 (1991), 157-165. 8. $\frac{}{240 .}$, Additive derivations of some operator algebras, Illinois J. Math. 35 (1991), 234-

9. __ Quadratic and quasi-quadratic functionals, Proc. Amer. Math. Soc. (to appear).

10. J. Vukman, $A$ result concerning additive functions in hermitian Banach $*$-algebras and an application, Proc. Amer. Math. Soc. 91 (1984), 367-372.

11. _- Some functional equations in Banach algebras and an application, Proc. Amer. Math. Soc. 100 (1987), 133-136.

DePARTMENT OF MATHEMATICS, UNIVERSity OF LJUbLJANA, JADRANSKA 19, 61000 LJUblJANA, SLOVENIA

E-mail address: peter.semrlQuni-1j.si 